\title{
SPR Method and Its Utilisation for Low Alcohols Concentrations Determination
}

\author{
Michal Lesňák ${ }^{1}$, František Staněk ${ }^{1}$, Ivo Hlavatý ${ }^{2}$, Jaromír Pištora ${ }^{3}$, Jan Procházka ${ }^{4}$ \\ ${ }^{1}$ Institute of Physics, Technical University of Ostrava, Ostrava, Czech Republic \\ ${ }^{2}$ Department of Mechanical Technology, Technical University of Ostrava, Ostrava, Czech Republic \\ ${ }^{3}$ Nanotechnology Centre, Technical University of Ostrava, Ostrava, Czech Republic \\ ${ }^{4}$ Institute of Mining Engineering and Safety, Technical University of Ostrava, Ostrava, Czech Republic \\ Email: michal.lesnak@vsb.cz, f.stanek@vsb.cz, ivo.hlavaty@vsb.cz, jaromir.pistora@vsb.cz, \\ jan.prochazka.st5@vsb.cz
}

Received 26 February 2015; accepted 13 March 2015; published 16 March 2015

Copyright (C) 2015 by authors and Scientific Research Publishing Inc.

This work is licensed under the Creative Commons Attribution International License (CC BY).

http://creativecommons.org/licenses/by/4.0/

(c) (i) Open Access

\begin{abstract}
SPR sensor with matrix arrangement of nanostructured golden spots enables both the study of low concentrations of substances in solution and the acquisition of information on the spatial layout of the diagnosed medium in solution. The linear response of this sensor was experimentally specified for standard solutions of alcohols in a concentration range of $0.01-0.5 \mathrm{wt} \%$. The influence of signal noise generated by carrier substance flowing through sensing dots has been removed by data processing. This approach enables to determine refractive index changes less than $3 \times 10^{-6}$.
\end{abstract}

\section{Keywords}

Plasmon Resonance, Alcohols, Concentration of Solutions

\section{Introduction}

A method of excitation of surface plasmons (Surface Plasmon Resonance-SPR) is one of the modern optical methods. In recent decades, advantages of this method have been used in the measurement of solid and liquid substances and in the study of various surfaces, thin layers and physical phenomena in them. SPR sensitivity to small changes in refractive index (up to $10^{-5}$ ) [1] is extraordinarily useful, especially in sensor applications, above all for very sensitive detectors of various substances in chemistry and biology (for instance, for the study of chemical reactions kinetics) [2].

The physical phenomenon of excitation of surface plasmons was observed by Wood [3] [4] already in the year 1902. It was Rayleigh [5] who began to study its physical nature; Fano [6], who explained it by means of 
excitation of electromagnetic surface waves, contributed significantly to the understanding of the phenomenon. The phenomenon was clarified completely by Otto [7] as late as the year 1968, and in the same year, Kretschmann and Raether [8] [9] described two different experimental configurations suitable for surface plasmon excitation. Since the end of the seventies of the last century, SPR has been used in the study of thin layers and phenomena at a metal-dielectric interface. Moreover, in the early eighties of the last century, this method began to be employed in biosensor design; at present, SPR-based sensors are widely used for the rapid identification of many biologically active substances, such as proteins, DNA, drugs, poisons, etc. [10] [11]. What seems to be a promise is the use of SPR in the area of thin layers and magneto-optical phenomena in them [12].

Surface plasmons as collective excitations of electrons bound to a conductor-insulator interface are considered [13]. The electromagnetic wave incident upon a medium containing a sufficient quantity of free charges, e.g. of electrons in metals (gold most frequently), reflects partially from this layer and penetrates partially into the medium below the conducting layer. The passed wave has the maximum intensity in the plane of the interface and its intensity decreases exponentially with perpendicular distance from the interface (designated as evanescent wave). Surface plasmons can be generated experimentally in a planar multilayer or grating structures. Under certain conditions, this wave can excite oscillations of free charges in this region.

Electromagnetic wave propagation in a metal is possible merely for frequencies lower than the plasmon frequency of the given metal [12]. For excitation of plasmon oscillations, polarized light (p-polarization) has to be used and the condition of equality of wave vectors of incident radiation and plasmon in the plane of metal-dielectric interface must be satisfied. Because the wave vector of light in air (vacuum) is smaller than the minimum critical wavelength necessary for the excitation of plasmons in the metal, plasmons cannot be excited by light incident directly from air.

For the excitation of surface plasmons, coupling prisms (Otto, Kretchmann-Raether configuration) or a periodic grating (see Figure 1) are used. In the first two variants, a gold layer is used most frequently.

At absolute reflection at the interface between an optically dense medium and an optically less dense medium, a surface (evanescent) wave can be excited by the origin of plasmons on the metal surface (Otto configuration, Figure 1(a)) or directly in a thin metal film by the excitation of electrons (Kretschmann-Raether configuration, Figure 1(b)). If a conducting layer is sufficiently thin (order of several ten $\mathrm{nm}$ ), surface plasmons can be excited at the farther interface of this layer (in the direction of incidence of the light wave in relation to the place of incidence). On the grating having the periodicity comparable to the wave length of the incident beam, diffraction occurs at the interface. As a result of interference, reflection and transmission modes and evanescence modes spreading along the interface are produced. The originated modes can excite surface plasmons (Figure 1).

The optimization of optical response of SPR sensors requires the tuning of two basic parameter sets, namely, optimization of sensor geometry and optimization of structural material selection [14]. For instance, in a paper [15], the authors achieve improvement in the response of the SPR structure by adding a dielectric nanolayer to the thin metallic layer. The same effect was obtained by the lamellar grating [16]. Especially in prism-coupled SPR systems at the interface between the coupling prism and the medium, in which the plasmon wave is excited, metal gratings with binary and cosine profiles are inserted [17]. Also geometrically more complex structures, e.g. so-called T-shaped and $\perp$-shaped lamellae [18], periodically arranged nanowires [19] and effective medium ap-

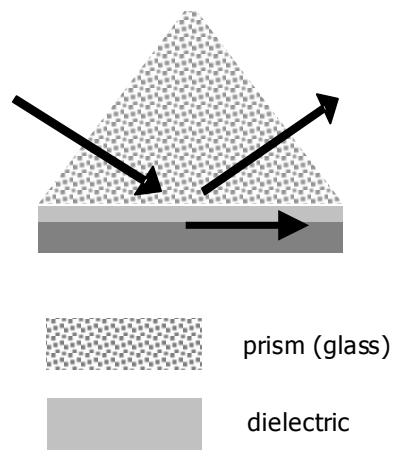

(a)
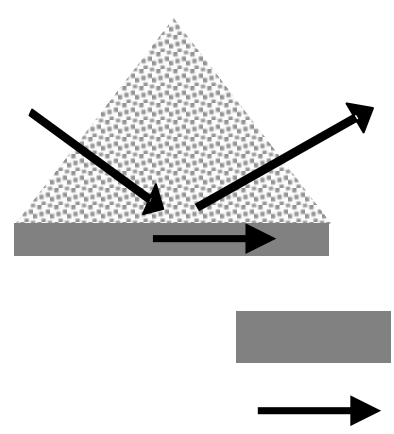

(b)

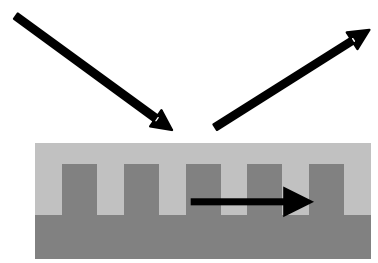

metal

surfaceplasmon

(c)

Figure 1. Experimental configuration for surface plasmon excitation: Otto configuration (a), Kretchmann-Raether configuration (b), and metal grating (c). 
proximation [20], have been applied.

The motivation of this paper is response limits determination of SPR sensors with matrix arrangement of nanostructured golden spots used for the specification of low alcohol concentration and the possibility to increase this sensitivity by data processing.

\section{Experimental Arrangement}

Surface plasmons are collective oscillations of noble metal electron gas, propagating along a metal and a dielectric interface. The excitation condition of the surface plasmon resonance (SPR) is very sensitive to changes of refractive index (the order of $10^{-5}-10^{-6}$ ) of the dielectric medium. A rise of SP wave results in reflection response from optical system as a dip in wavelength or angular spectrum of reflected beam. Thus, the location, shape, depth or shift of this minimum can serve as detection phenomena used in a sensing device.

For testing of alcohols concentration measurement, an instrument SPRi-Lab+TM from the company JY-Horiba (Genoptics-Horiba Scientific, Orsay, France) was used. The device is based on the reflectivity measurement of monochromatic p-polarized incident light $(635 \mathrm{~nm})$ at the fixed angle of incidence. This makes it possible to obtain very rapidly a sensogram (reflectivity change depending on time). During experiment, the Kretschmann configuration is applied.

In the experiment the SPRi instrument was also used for the observation of influence of alcohol concentration response. A chip with only a gold layer was applied. The used carrier liquid was demineralized water. To this carried liquid, individual measured samples of diagnosed substances were added by means of a $200 \mu$ loop. Measurements were carried out at a temperature of $20^{\circ} \mathrm{C}$. The temperature was maintained by means of a Thorlabs controller. For transporting the liquid, a peristaltic pump (Minipuls 3M312, Gilson) was used; the flow rate of the liquid was set at $50 \mu \mathrm{l} / \mathrm{min}$.

\section{Measurements}

The measurements were carried out on ten samples of solutions in the following order: glycerol, ethanol, isopropanol, isobutanol, n-butanol, methanol, n-hexanol, ethylene glycol, secondary butanol and tertiary butanol. Each sample was measured five times under the same conditions. Based on this repetition, an estimate of uncertainty was calculated. The concentrations of the tested samples were selected as follows (in grams of substance per 100 grams of solution, in wt per cent): $0.5 ; 0.2 ; 0.1 ; 0.075 ; 0.05 ; 0.025 ; 0.01$ (injection of the tested substance to the measuring cell). Appropriate quantities of alcohol were weighted on an analytical balance (ADAM from Schoeller) with responsibility of $0.0001 \mathrm{~g}$. Solutions were formed by demineralized water and appropriate p.a. quality alcohol.

The experiments have been realized at the optimum angle of incidence on the prism. A specifically measured sensogram is given in Figure 2. At the beginning, calibration was done, and then appropriate measurements were realized. During the experiments the software supplied by Genoptics-Horiba Scientific was applied. MS Excel and Matlab programs were used for processing.

\section{Results}

Experimental results for glycerine are illustrated in Figure 2. The response peaks in reflectivity related to different glycerine concentration are modulated by practically linear reflectivity-time dependence created by carrier substance influence. Experimental data were signal-processed to remove the influence of the carrier substance flowing through (final effect is shown in Figure 3). At the beginning, there is a region of process stabilization. The graph in Figure 4 documents the linear dependence of signal response on concentration for measured samples. Obtained results for the whole set of tested substances are summarised in Table 1.

\section{Conclusions}

The linear response of the SPR detector was specified on standard solutions of alcohols in a concentration range of $0.01-0.5 \mathrm{wt} \%$. The relevant slope of this line depends on the number of carbons and the structure of alcohol and on the refractive index of the liquid. Of tested substances, the smallest line slope was obtained for methanol; on the contrary, the used method proved to be the most sensitive in the case of hexanol (roughly one order difference). From Figure 2, the experimental setup used by us reacts unambiguously to a glycerine concentration 


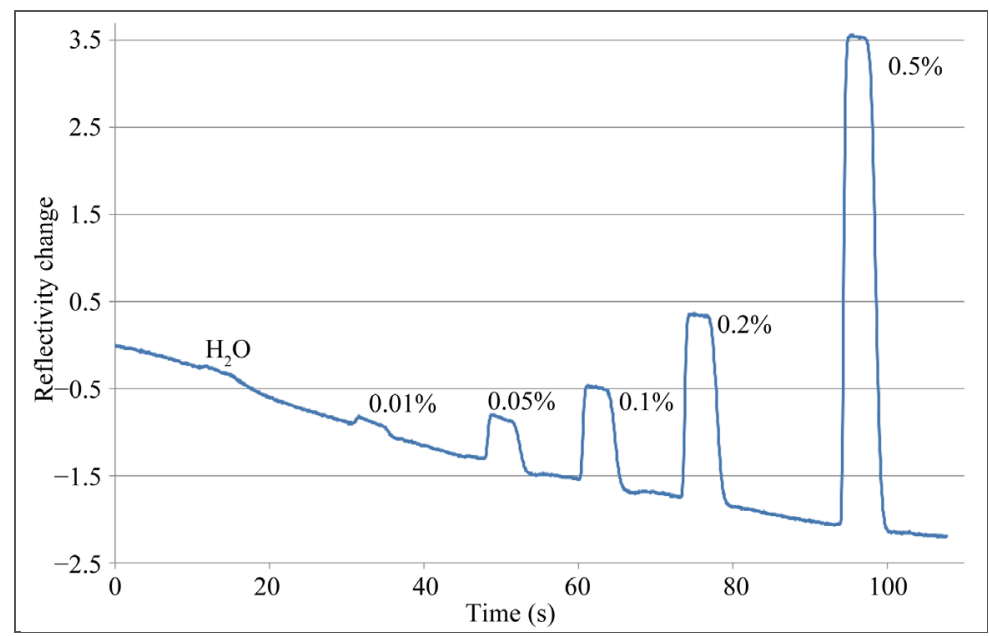

Figure 2. Dependence of reflectivity change on concentration for glycerine.

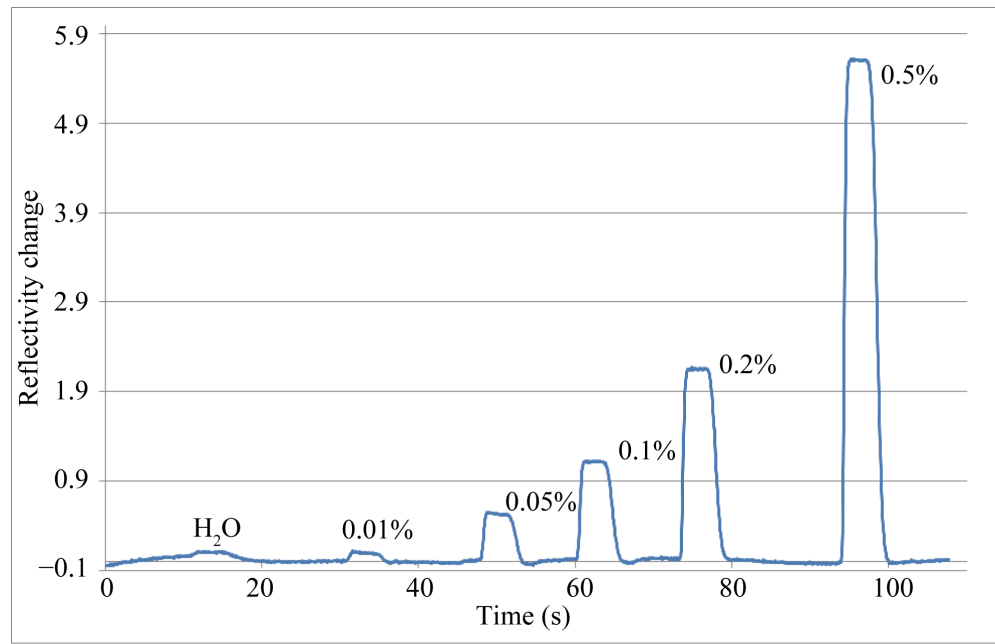

Figure 3. Dependence of reflectivity change on concentration for glycerine measurement (after background computation).

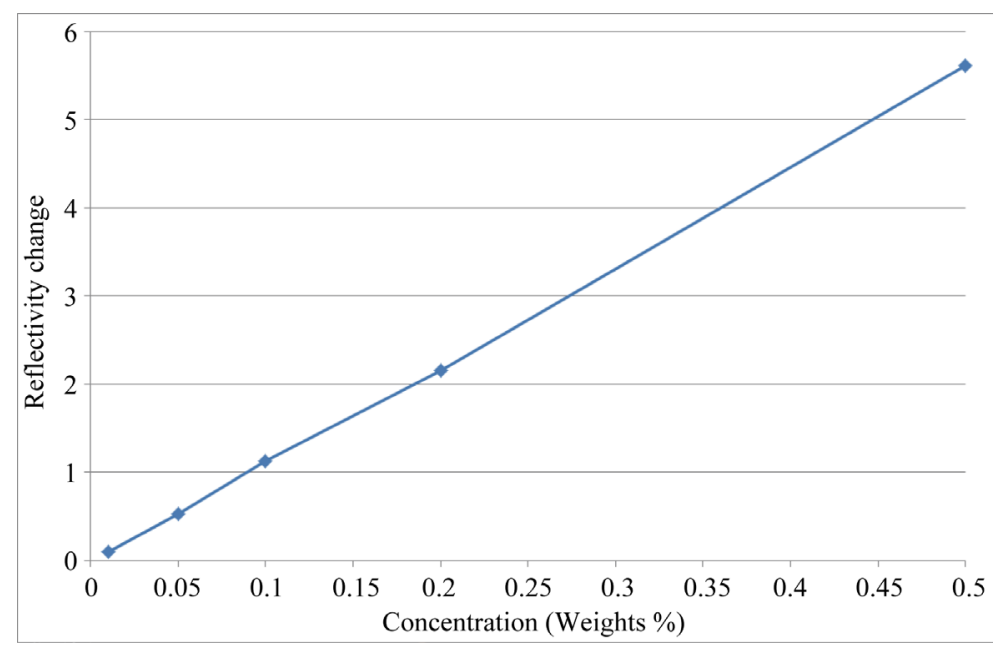

Figure 4. Dependence of reflectivity change on concentration for glycerine measurement. 
Table 1. Measured parameters of alcohol concentrations.

\begin{tabular}{ccccccc}
\hline & $\mathrm{a}$ & $\mathrm{b}$ & $\operatorname{tg} \beta\left[^{\circ}\right]$ & $\begin{array}{c}\text { Correlation } \\
\text { coefficient }\end{array}$ & $\begin{array}{c}\text { Estimation of } \\
\text { uncertainty }\end{array}$ & Refractive index \\
\hline Methanol & -0.015 & 1.54 & 57.04 & 0.994 & 0.038 & 1.329 \\
Ethanol & 0.10 & 5.02 & 78.73 & 0.997 & 0.070 & 1.3611 \\
Isopropanol & -0.013 & 5.62 & 79.96 & 0.997 & 0.073 & 1.377 \\
Glycerol & -0.029 & 11.23 & 84.91 & 0.999 & 0.041 & 1.4593 \\
N-butanol & 0.0014 & 8.42 & 83.32 & 0.999 & 0.032 & 1.3973 \\
Isobutanol & 0.074 & 7.61 & 82.51 & 0.999 & 0.056 & 1.39908 \\
Secondary butanol & -0.011 & 8.56 & 83.33 & 0.999 & 0.079 & 1.3761 \\
Tertiary butanol & -0.10 & 7.75 & 82.64 & 0.998 & 0.092 & 1.3671 \\
Ethylene glycol & -0.025 & 7.61 & 82.51 & 0.999 & 0.035 & 1.43854 \\
N-hexanol & -0.16 & 17.69 & 86.76 & 0.999 & 0.15 & 1.4158 \\
\hline
\end{tabular}

of $0.01 \%$. In the solution applied, this corresponds to a change in the refractive index of $3 \times 10^{-6}$. This value is in accordance with the data published for the design of serial and parallel sensors based on SPR for the analysis of liquid substances [12]. It appears that a matrix arrangement of thin layer areas plated with gold on a nanoscale as an SPR sensor enables both the study of low concentrations of substances in solution and the acquisition of information on the spatial layout of the diagnosed medium in solution. The mentioned geometry of the SPR sensor is a way towards the increased sensitivity of measurement equipment based on plasmon waves for the study of alcohols' concentration in the given solution.

The SPR method is used for the detection and identification of the given alcohol in solution and for its quantification thanks to linear response dependence on concentration. A disadvantage is the need to know the kind of the alcohol that is tested in the carrier liquid. We try to remove this disadvantage by using of ATR method (FTIR-Fourier Transform Infrared Spectroscopy). We believe that we shall be able not only to determine low alcohol concentrations but also to identify the kind of alcohol.

\section{Acknowledgements}

This paper was created in the Project No. LO1203 "Regional Materials Science and Technology Centre-Feasibility Program" funded by Ministry of Education, Youth and Sports of the Czech Republic.

\section{References}

[1] Raether, H. (1988) Surface Plasmons on Smooth and Rough Surfaces and on Gratings. Springer, Berlin.

[2] Homola, J., Ed. (2006) Surface Plasmon Resonance Based Sensors. Springer, New York. http://dx.doi.org/10.1007/b100321

[3] Wood, R.W. (1902) Philosophical Magazine Series 6, 4, 396-402. http://dx.doi.org/10.1080/14786440209462857

[4] Wood, R.W. (1912) Philosophical Magazine Series 6, 23, 310-317. http://dx.doi.org/10.1080/14786440208637224

[5] Rayleigh, L. (1907) Proceedings of the Royal Society of London, Series A, 79, 399-415. http://dx.doi.org/10.1098/rspa.1907.0051

[6] Fano, U. (1941) Journal of the Optical Society of America, 31, 213-222. http://dx.doi.org/10.1364/JOSA.31.000213

[7] Otto, A. (1968) Zeitschrift für Physik, 216, 398-410. http://dx.doi.org/10.1007/BF01391532

[8] Kretschmann, E. and Raether, H. (1968) Zeitschrift für Naturforschung, Teil A, 23, 2135-2136.

[9] Kretschmann, E. (1971) Zeitschrift für Physik, 241, 313-324. http://dx.doi.org/10.1007/BF01395428

[10] Schasfoort, R.B.M. and Tudos, A.J., Eds. (2008) Handbook of Surface Plasmon Resonance. RSC Publishing, ISBN: 978-0-85404-267-8. http://dx.doi.org/10.1039/9781847558220

[11] Liedberg, B., Nylander, C. and Lundstorm, I. (1983) Sensors and Actuators, 4, 299-304. http://dx.doi.org/10.1016/0250-6874(83)85036-7 
[12] Lesňák, M., Vlček, J. and Pištora, J. (2011) Hutnické listy, Praha: Ocelot, LXIV, č. 3, 76-79, ISSN: 0018-8069.

[13] Kretschmann, E. and Raether, H. (1968) Zeitschrift für Naturforschung A, 23, 2135-2136.

[14] Halagacka, L., Vanwolleghem, M., Postava, K., Dagens, B. and Pistora, J. (2013) Optics Express, 21, $21741-21755$. http://dx.doi.org/10.1364/OE.21.021741

[15] Iwata, T. and Komoda, G. (2008) Applied Optics, 47, 2386-2391 http://dx.doi.org/10.1364/AO.47.002386

[16] Abdulhalim, I. (2009) Journal of Optics A: Pure and Applied Optics, 11, Article ID: 015002. http://dx.doi.org/10.1088/1464-4258/11/1/015002

[17] Wu, B. and Wang, Q.K. (2008) Chinese Physics Letters, 25, 1668-1671. http://dx.doi.org/10.1088/0256-307X/25/5/040

[18] Alleyne, C.J., Kirk, A.G., McPhedran, R.C., Nicorovici, N.A.P. and Maystre, D. (2007) Optics Express, 15, $8163-8169$. http://dx.doi.org/10.1364/OE.15.008163

[19] Byun, K.M., Kim, S.J. and Kim, D. (2005) Optics Express, 13, 3737-3742. http://dx.doi.org/10.1364/OPEX.13.003737

[20] Foldyna, M., Postava, K., Ossikovski, R., De Martino, A. and Garcia-Caurel, E. (2006) Journal of the European Optical Society, 1, 06015-1-06015-9. 